\title{
Caracterización de los signos clínicos del Síndrome Combinado de Kelly de un hogar geriátrico de la ciudad de Bucaramanga
}

\author{
Characterization of the clinical signs of the Combined Kelly Syndrome, in the geri- \\ atric institution in Bucaramanga
}

Ligia Lizeth Blanco Villamizar' ${ }^{1}$, Wilmer Francisco Capacho Vera' ${ }^{1}$ Jairo Fernando Picón Peña ${ }^{2}$

Para citar este artículo: Blanco LL, Capacho WF, Picón JF. Caracterización de los signos clínicos del síndrome combinado de Kelly en el hogar geriátrico 'Luz de Esperanza' de la ciudad de Bucaramanga. Ustasalud 2016;15:6-11

\section{RESUMEN}

Licencia Creative Commons

\section{(c) $(1)(9)$} lo tanto, los lectores pueden acceder libremente a los artículos en su formato .pdf, igualmente podrán descargarlos y difundirlos; sin embargo no podrán modificarlos o alterarlos, adicionalmente se debe reconocer la autoría de las personas que figuran en las publicaciones, pero estas no podrán comercializadas.

\begin{abstract}
1 Facultad de Odontología, Universidad Santo Tomás, Bucaramanga

${ }^{2}$ Grupo de Investigación Sistema Estomatognático y Morfofisiología - SEMF, Tecnología de Laboratorio Dental, Universidad Santo Tomás, Bucaramanga
\end{abstract}

Autor de correspondencia: Jairo Fernando Picón Peña Correo electrónico: jairof.picon@ustabuca.edu.co
Objetivo: establecer la frecuencia de los signos del Síndrome Combinado de Kelly (SCK) de un hogar geriátrico de la ciudad de Bucaramanga.

Materiales y métodos: se realizó un estudio observacional descriptivo de corte transversal. La muestra la constituyeron 79 personas a quienes se les aplicó un instrumento y posteriormente fueron evaluados mediante un examen clínico intraoral para determinar la presencia de los signos según los criterios del instrumento. El análisis estadístico se realizó en el programa Stata/MP 14.0 que comprendió un análisis descriptivo y bivariado donde se ejecutaron pruebas como $\mathrm{Chi}^{2}$ y el test exacto de Fisher, según correspondiera, con el fin de estudiar la relación de las variables.

Resultados: se encontró que el 52,7\% de los participantes presentaron cinco signos del SCK.

Conclusión: más de la mitad de los participantes presentaron signos del SCK, encontrádose como factores asociados la presencia de prótesis total en el maxilar superior, la ausencia de prótesis en el maxilar inferior y el edentulismo parcial en el maxilar inferior.

Palabras clave: síndrome combinación, síndrome combinado de Kelly, síndrome de hiperfunción anterior.
Recibido para publicación 19 de febrero de 2016. Aceptado para publicación: 05 de septiembre de 2016.

\section{ABSTRACT}

Objective: to establish the frequency of the CKS signs of the a geriatric institution from Bucaramanga.

Materials and methods: a cross-sectional, observational, descriptive study was executed. The sample consisted of 79 individuals who were given an instrument and then evaluated through an intraoral clinical examination to determine the presence of signs according to the criteria considered in the format. Statistical analysis was performed in the Stata / IC 14.0 software, which included a descriptive and bivariate analysis where $\mathrm{Chi}^{2}$ and Fisher exact tests were used, as appropriate, in order to study the relationship of the variables. Results: it was found that $52.7 \%$ of the participants presented the five signs of SCK.

Conclusion: more than half of the participants presented signs of SCK. Associated factors were the presence of total prosthesis in the upper jaw, the absence of prosthesis in the lower jaw and partial edentulism in the lower jaw.

Keywords: combination syndrome, Kelly syndrome, hyperfunction syndrome. 


\section{INTRODUCCIÓN}

El Síndrome Combinado de Kelly (SCK) ${ }^{1}$ es la evolución en el tiempo de condiciones patológicas del sistema estomatognático ${ }^{2}$.

El glosario de términos de prostodoncia ${ }^{3}$ en el 2005 define el término SCK como el rasgo particular que se ocasiona cuando los pacientes se encuentran edéntulos en el maxilar superior y antagonizan con dientes antero inferiores naturales, de allí se manifiestan diversas características clínicas, como son: reabsorción ósea en la región anterior del maxilar, sobrecrecimiento tuberosidad, hiperplasia papilar del paladar, la extrusión de los dientes de la mandíbula natural restante y reabsorción ósea mandibular posterior, este término lo introdujo el doctor Ellswoth Kelly ${ }^{1}$ en 1972. Este síndrome es también llamado, síndrome de hiperfunción anterior y síndrome de combinación ${ }^{4,5}$.

Años después, Saunders y colaboradores ${ }^{6}$, en 1979, observaron nuevas características afines con el SCK, como lo son: pérdida de dimensión vertical oclusal, discrepancia en el plano oclusal, reposicionamiento espacial de la mandíbula, pobre adaptación de la prótesis, épulis fisuratum y cambios periodontales (Figura 1); no obstante estos cambios no están generalmente presentes en todos los casos con el SCK.

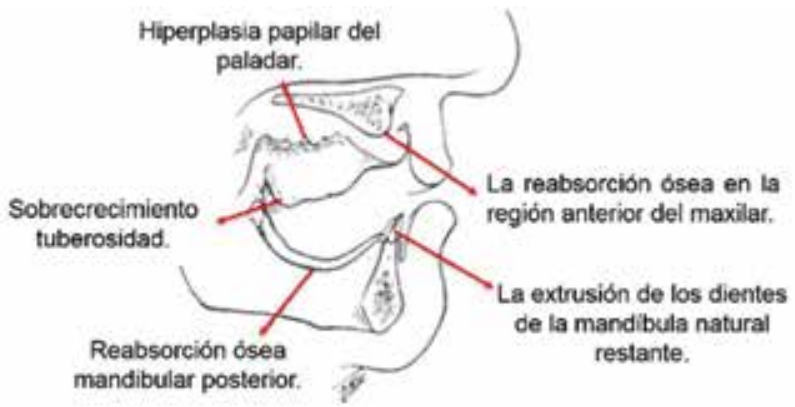

Fuente: Saunders, D.D.S (1979) the maxillary complete denture opposing the mandibular bilateral distal-extensión partial denture: treatment considerations ${ }^{6}$.

Figura 1. Signos de SCK.

El SCK inicia con la pérdida de dientes debido a caries, enfermedad periodontal, trauma, defectos congénitos y tratamientos iatrogénicos. La pérdida dental tiene un impacto negativo en la masticación, fonación, deglución y estética, afectando la calidad de vida del individuo. Después de la extracción, el hueso alveolar residual comienza un período de reabsorción rápida durante aproximadamente diez semanas, seguido por una reabsorción más lenta pero progre$\operatorname{siva}^{5,7}$ (Figuras 2-6).

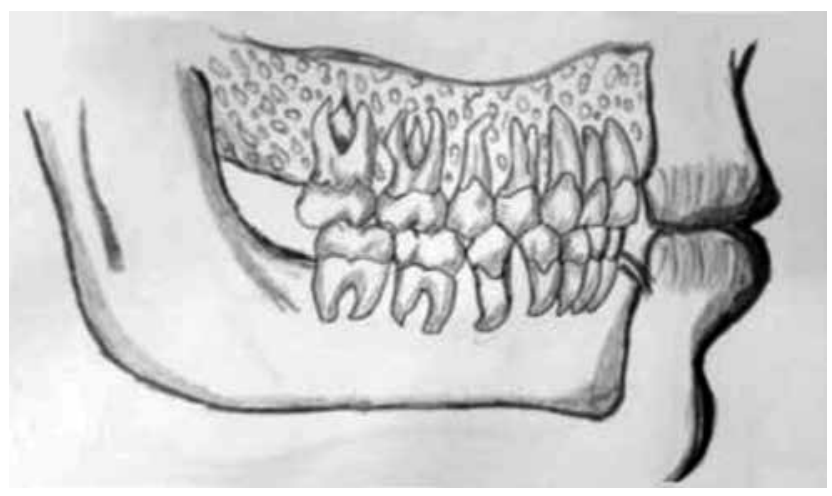

Figura 2. Paciente sin alteración.

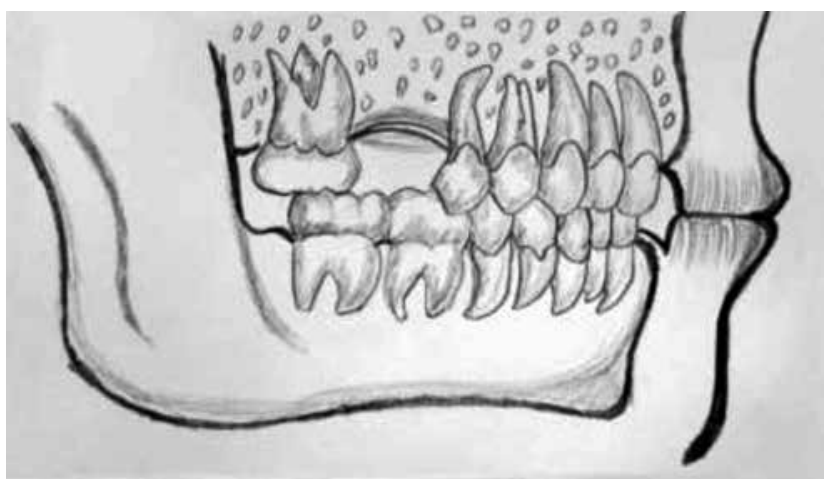

Figura 3. Progresión de los signos del SCK.

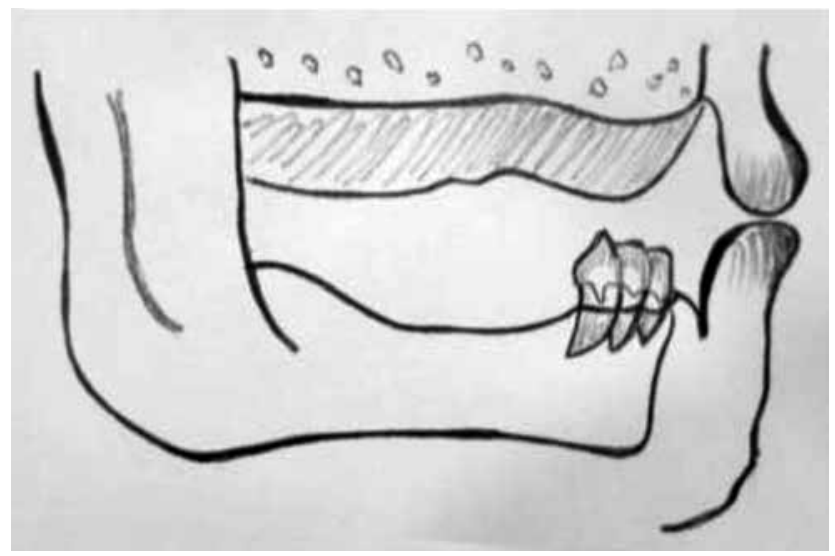

Figura 4. Progresión de los signos del SCK. 


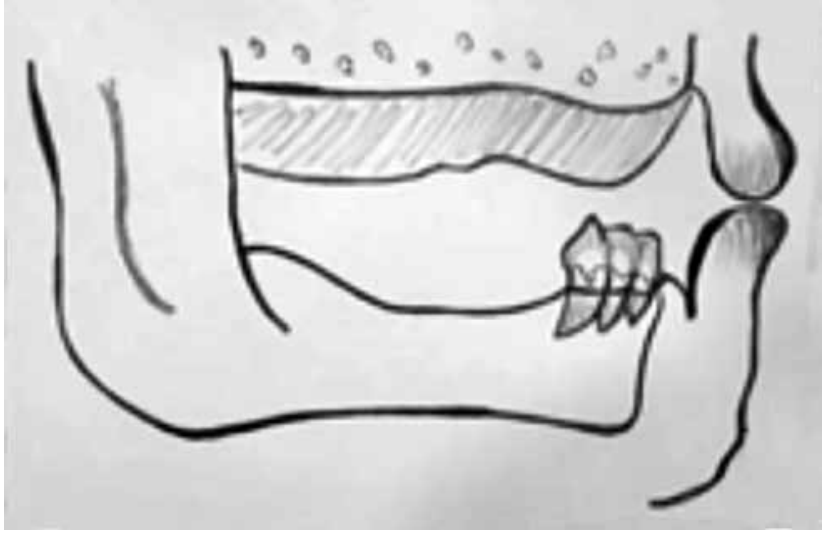

Figura 5. Progresión de los signos del SCK.

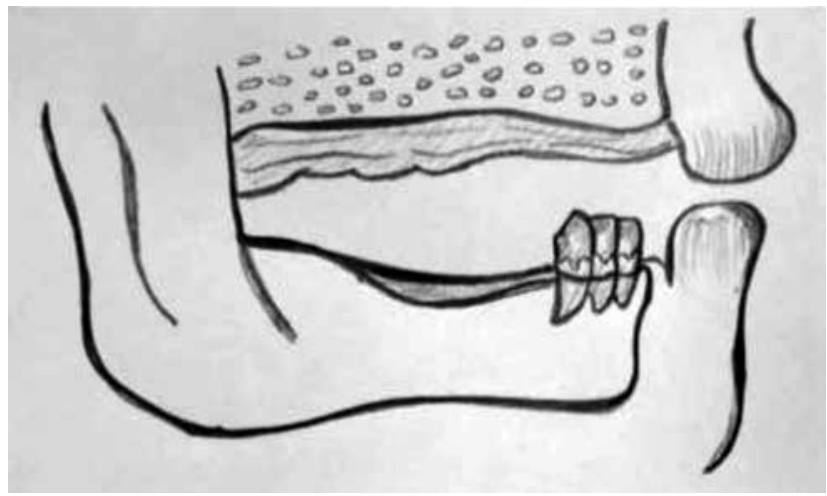

Figura 6. Progresión de los signos del SCK.

Fuente: autores.

En Colombia el ENSAB IV ${ }^{8}$ identificó que a los 15 años de edad un $15 \%$ de los adolescentes presentaban pérdida del algún diente y que la cantidad de dientes perdidos incrementa a medida que se aumenta la edad. El $11 \%$ de la población ha perdido la totalidad de sus dientes superiores, situación que se evidencia con el uso de prótesis total y prótesis parcial removible en la población nacional. De la población edéntula el $32 \%$ actualmente utiliza algún tipo de tratamiento de rehabilitación. La población en edades comprendidas entre los 65 y 79 años utilizan prótesis con una prevalencia de uso del $77 \%$.

Comprendiendo la evolución que presenta la enfermedad oclusal, Guerrero y Cols. sugieren que el SCK es una posible etapa avanzada de un síndrome de colapso de mordida posterior, si este no se diagnostica apropiadamente ni se trata de manera adecuada ${ }^{9}$. A nivel regional y nacional no existe ningún estudio que relacione los signos clínicos del SCK y su asiduidad en la población. Por lo anterior, el objetivo del presente estudio fue establecer la frecuencia de los signos del SCK del hogar geriátrico 'Luz de Esperanza' de la ciudad de Bucaramanga, apoyados en la investigación del doctor Ellswoth Kelly ${ }^{1}$ acerca del SCK.

\section{MATERIALES Y MÉTODOS}

Se realizó un estudio observacional descriptivo de corte transversal. La población fue la totalidad de las personas residentes del hogar geriátrico 'Luz de Esperanza' de Bucaramanga. Considerando que el universo integró la muestra del total de la población, no se implementó un muestreo, pero se consideraron criterios de selección: se incluyeron las personas que el día del examen clínico estuvieron presentes y se excluyeron aquellas que presentaron trismus, las que se negaron al examen clínico intraoral o que durante la realización del mismo se reusaron a permitir terminarlo. Se contó con el consentimiento informado para cada participante y firmado por los cuidadores del hogar geriátrico 'Luz de Esperanza' de la ciudad de Bucaramanga, previamente revisado y aprobado por el Comité de Investigación de la Universidad Santo Tomás, según lo avalado por el Comité de Ética Institucional y de acuerdo con la Resolución No.008430 del Ministerio de Salud de Colombia del 4 de octubre de $1993^{10}$.

\section{Recolección de información}

Después de diligenciar el consentimiento informado, se sometió a los participantes a un examen oral clínico por parte de la investigadora capacitada y calibrada para hacer el examen diagnóstico, quien realizó una evaluación diagnóstica para determinar la presencia de los signos del SCK, tal como describe Kelly ${ }^{1}$ : reabsorción ósea en la región anterior del maxilar, sobrecrecimiento de la tuberosidad, hiperplasia papilar del paladar, la extrusión de los dientes de la mandíbula natural restante y reabsorción ósea mandibular posterior (Tabla 1$)^{2,3}$. 
Tabla 1. Signos del SCK

\begin{tabular}{ll}
\hline Características del SCK & Evaluación clínica \\
\hline $\begin{array}{l}\text { La reabsorción ósea en la región } \\
\text { anterior del maxilar }\end{array}$ & $\begin{array}{l}\text { Se observó tejido flácido en la re- } \\
\text { gión anterior de la cresta residual } \\
\text { susceptible de desplazamiento. }\end{array}$ \\
$\begin{array}{l}\text { Sobrecrecimiento tuberosidad } \\
\text { Crecimiento horizontal de tejido } \\
\text { fibroso o de la médula en la de- } \\
\text { recha. }\end{array}$ \\
$\begin{array}{l}\text { Hiperplasia papilar del paladar } \\
\text { La extrusión de los dientes de la } \\
\text { mandíbula natural restante }\end{array}$ & $\begin{array}{l}\text { Se observó desgaste a nivel del es- } \\
\text { paladar duro o de la dentina. }\end{array}$ \\
$\begin{array}{l}\text { Reabsorción ósea eritematosa } \\
\text { posterior }\end{array}$ & $\begin{array}{l}\text { Se observó reabsorción ósea acen- } \\
\text { tuada en la región posterior edén- } \\
\text { tula. }\end{array}$ \\
\hline
\end{tabular}

El investigador con especialidad en Rehabilitación Oral resolvía cualquiera de los casos en los que se presentaba disparidad en la identificación de los signos característicos del síndrome.

\section{Análisis estadístico}

Posterior a la recolección de los datos, se realizó su digitalización por duplicado en bases de datos de Microsoft Corporation Excel. 2015 United States. Para el análisis estadístico se utilizó el programa estadístico Stata (Statistical Software Stata/MP Release 14. 2015. College Station; TX: United States). Se estableció que para este estudio todos los valores con $\mathrm{p}<0,05$ eran estadísticamente significativos.

\section{RESULTADOS}

La muestra estudiada fue de 79 personas para las características sociodemográficas, 5 de éstas fueron excluidas durante el diligenciamiento del instrumento. Así, la muestra definitiva para el examen clínico oral fue de 74 participantes.

El presente estudio evaluó 79 personas residentes del hogar geriátrico 'Luz de Esperanza' de la ciudad de Bucaramanga, con un promedio de edad de $74 \pm 15,2$ años, donde el $60,8 \%(n=48)$ de los participantes eran del género masculino.
De la muestra estudiada para el examen clínico oral se encontró que en el maxilar superior el 50\% ( $n=37)$ de los participantes utilizaban prótesis total, en contraste con un $32,5 \%(n=24)$ que no portaban ninguna prótesis en este maxilar. De los 24 participantes que no utilizaban prótesis, 19 que representan el 79,2\% eran edéntulos totales.

Los pacientes no fueron evaluados en relación con el diagnóstico SCK contando signos específicos para determinar si tenían el síndrome o no, en cambio sí se realizó con respecto a la presencia o ausencia de cada signo y el número de signos por individuo. De las características del SCK un 74,3\% $(\mathrm{n}=55)$ presentó reabsorción ósea en la región anterior del maxilar, el 64,9\% $(\mathrm{n}=48)$ presentó sobrecrecimiento de la tuberosidad (Tabla 2).

Tabla 2. Los signos del SCK detectados en el estudio

\begin{tabular}{lcc}
\hline \multirow{2}{*}{ Característica del SCK } & \multicolumn{2}{c}{ Categoría } \\
\cline { 2 - 3 } & \multicolumn{1}{c}{ Sí } & No \\
\hline $\begin{array}{l}\text { La reabsorción ósea en la región anterior } \\
\text { del maxilar }\end{array}$ & $55(74,3)$ & $19(25,7)$ \\
\hline $\begin{array}{l}\text { Sobre crecimiento tuberosidad } \\
\text { Hiperplasia papilar del paladar }\end{array}$ & $48(64,9)$ & $26(35,1)$ \\
\hline $\begin{array}{l}\text { La extrusión de los dientes de la mandí- } \\
\text { bula natural restante }\end{array}$ & $42(59,5)$ & $30(40,5)$ \\
\hline $\begin{array}{l}\text { Reabsorción ósea mandibular posterior } \\
\text { ne }\end{array}$ & $39(52,7)$ & $32(43,2)$ \\
\hline
\end{tabular}

Cuando se examinaron los signos del SCK, se encontró que el 52,7\% $(n=39)$ de los participantes evaluados en el examen clínico mostraron 5 signos del SCK y el 25,7\% no registró ningún signo clínico. En cuanto al número de signos identificados por pacientes se encontró una mediana de 3,5.

Con relación a la condición bucal en el maxilar superior e inferior (Tabla 3), con los signos del SCK (Tabla 4) no se evidenciaron diferencias estadísticamente significativas según el género $(\mathrm{p}<0,05)$. Por otra parte, al analizarse el tipo de prótesis en el maxilar superior con la presencia de los signos del SCK, se encontró que la mayoría de pacientes que presentaron algún signo característico del síndrome eran portadores de prótesis total, se observaron diferencias estadísticamente significativas $(p<0,0001)$. 
En cuanto a los participantes que no eran portadores de prótesis en el maxilar inferior en su mayoría presentaron los signos del SCK, estas diferencias fueron estadísticamente significativas $(\mathrm{p}<0,0001)$.

De los participantes edéntulos parciales en el maxilar inferior en su mayoría presentaron los signos del SCK, estas diferencias fueron estadísticamente significativas $(p<0,05)$ en cuatro signos excepto en el signo de aumento fibroso de las tuberosidades.

\section{DISCUSIÓN}

En una revisión de literatura nacional y regional no se encontraron estudios epidemiológicos del $\mathrm{SCK}^{11}$. En consecuencia, en el presente estudio, la frecuencia de los signos del SCK se evaluó en lugar del diagnóstico.

En el presente estudio, 74,3\% $(\mathrm{n}=55)$ de la muestra mostró reabsorción ósea en la región anterior del maxilar, el signo clínico SCK más frecuente entre las variables dependientes estudiadas (Tabla 2). Esta característica clínica es común entre estos pacientes; debido a la extracción de los dientes, inicia un proceso de remodelación del hueso alveolar, incluyendo la reabsorción ósea y un cambio en el contorno, la prótesis superior entonces se mueve hacia arriba en la región anterior y abajo en la región posterior durante la función. La frecuencia alta de este signo es de conformidad con las conclusiones de Kelly ${ }^{1,12}$.

Por otra parte al analizarse el tipo de prótesis en los maxilares según la presencia de los signos del SCK, se observaron diferencias estadísticamente significativas $(p<0,0001)$ en los participantes que en el maxilar superior utilizaban prótesis total superior y en el maxilar inferior no portaban tratamiento rehabilitador, ya que este grupo presentó algunos de los cinco signos del SCK ${ }^{13}$.

Un crecimiento excesivo de las tuberosidades estuvo presente en el 64,9\% de la muestra. Shen y Gongloff $^{14}$ encontraron una frecuencia similar del 56\% cuando se evaluaron los pacientes clase I de Kennedy Applegate que utilizaron prótesis total superior y prótesis parcial removible inferior.
El signo del SCK menos frecuente fue la pérdida ósea alveolar y altura por debajo de la cresta mandibular posterior (52,7\%). Esta característica clínica es debido al proceso de resorción ósea mandibular natural que se produce principalmente en la altura ${ }^{15}$. Tolstunov ${ }^{16}$ expresó que el hueso se deposita y se reabsorbe en función de las tensiones que se les plantean ${ }^{17}$.

Una de las limitaciones de este estudio fue el hecho de que el muestreo no fue probabilístico y los pacientes tampoco fueron escogidos aleatoriamente.

A pesar de las limitaciones, el gran número de signos característicos presentes en esta investigación es un hecho que llama la atención, resultante de la falta de seguimiento a los pacientes portadores de prótesis removibles. Esta situación cambiará cuando el odontólogo que trata al paciente entienda la importancia de la prevención, el diagnóstico y la intervención.

\section{CONCLUSIÓN}

Más de la mitad de los participantes presentaron cinco signos del SCK. La presencia de prótesis total en el maxilar superior, la ausencia de prótesis en el maxilar inferior y el edentulismo parcial en el maxilar inferior se asociaron con la presencia de los signos SCK. La falta de estudios epidemiológicos nacionales y regionales, y la falta de conocimiento acerca del diagnóstico clínico oral de los signos del SCK en la población le han impedido lograr la plena aceptación como síndrome médico. Sin embargo, como muestra este estudio, no se puede ignorar la presencia de los signos individuales asociados al SCK. Con el fin de poder decir cuántos años son necesarios para que el SCK ocurra en pacientes edéntulos, se debe planificar un estudio de seguimiento para observar a los pacientes desde el momento en que se convirtieron en edéntulos. De esta manera, los signos relacionados con el SCK pueden ser confirmados por investigaciones futuras, incluyendo estudios clínicos a largo plazo.

\section{BIBLIOGRAFÍA}

1. Kelly E. Changes caused by a mandibular removable partial denture opposing a maxillary complete denture. J Prosthet Dent. 1972;27(2):140-150. doi: https://doi. org/10.1016/0022-3913(72)90190-4. 
2. Palmqvist, S, Carlsson, GE. The combination syndrome: A literature review. J Prosthet Dent. 2003;90(3):270-5. doi:https://doi.org/10.1016/S0022-3913(03)00471-2.

3. Academy of Prosthodontics. The glossary of terms. 2005;94(1):10-92.

4. Shen K, Gongloff RK. Prevalence of the 'combination syndrome' among denture patients. J Prosthet Dent. 1989;62(6):642-4. doi:https://doi. org/10.1016/0022-3913(89)90582-9.

5. Carlsson GE. Measurements on casts of the edentulous maxilla. Odontol Revy. 1966;17(4):386-402.

6. Saunders TR, Gillis RE Jr, Desjardins RP. The maxillary complete denture opposing the mandibular bilateral distal-extension partial denture: Treatment considerations J Prosthet Dent. 1979;41(2):124-8.doi: https://doi. org/10.1016/0022-3913(79)90292-0.

7. Wyatt CC. The effect of prosthodontic treatment on alveolar bone loss: a review of the literature. J Prosthet Dent. 1998;80(3):362-366. doi:https://doi.org/10.1016/ S0022-3913(98)70138-6.

8. ENSAB IV, Ministerio de Salud y Protección Social. IV Estudio nacional de salud bucal, situación en salud bucal. 2014. [Internet] Recuperado a partir de: https://www. minsalud.gov.co/sites/rid/Lists/BibliotecaDigital/RIDE/ VS/PP/ENSAB-IV- Situacion-Bucal-Actual.pdf.

9. Guerrero C, Martín D, Galvis A. Evolución de la patología oclusal: Una revisión de la literatura. J Oral Res. 2013;2(2):77-85. doi: 10.17126/JORALRES.2013.017.

10. Ministerio de Salud, Colombia. Resolución 8430 de 1993.

11. Rojas Molano L, Galvis A, Marín D. Contrastación de los hallazgos y diagnósticos oclusales reportados en la lite- ratura, con los registrados en las historias clínicas de pacientes atendidos en las clínicas del adulto de la Facultad de Odontología de la Universidad Nacional de Colombia. [Trabajo de grado Rehabilitación Oral] Colombia: Universidad Nacional de Colombia, 2014.

12. Souza Lima FSLC, Vanzillotta PS, Ferreira CA, Monteiro Briote MI. Síndrome da Combinação. Rev. bras. odontol. Rio de Janeiro. 2012;69(2):199-202. [Internet] Recuperado a partir de: http://revodonto.bvsalud.org/scielo.php?script=sci_arttext\&pid=S0034-72722012000200012\&ln$\mathrm{g}=\mathrm{pt}$.

13. Barber HD, Scott RF, Maxson BB, Fonseca RJ. Evaluation of anterior maxillary alveolar ridge resorption when opposed by the transmandibular implant. J Oral Maxillofac Surg. 1990;48(12):1283-128. doi: https://doi. org/10.1016/0278-2391(90)90483-I.

14. Shen K, Gongloff RK. Prevalence of the 'combination syndrome' among denture patients. J Prosthet Dent. 1989;62(6):642-644. doi: https://doi. org/10.1016/0022-3913(89)90582-9.

15. Xie Q, Närhi TO, Nevalainen JM, Wolf J, Ainamo A. Oral status and prosthetic factors related to residual ridge resorption in elderly subjects. Acta Odontol Scand. 1997;55(5):306-313. doi: https://doi. org/10.3109/00016359709114969.

16. Tolstunov L. Management of biomechanical complication of implant-supported restoration of a patient with combination syndrome: a case report. J Oral Maxillofac Surg. 2009;67(1):178-88. doi: https://doi.org/10.1016/j. joms.2008.09.013.

17. Ramiro Guillén C, Guevara García C, Gómez Polo M, Celemín Viñuela A. ¿Qué tratamiento podemos elegir para rehabilitar pacientes con Síndrome de Combinación? Cient Dent. 2011;8(3):65-69. 